\title{
IVF Outcomes of Microdose Flare-up, GnRH Antagonist, and Long Protocols in Patients having a Poor Ovarian Response in the First Treatment Cycle
}

\author{
Erhan Demirdağ ${ }^{1}$, M. Funda Cevher Akdulum ${ }^{1}$, Ismail Guler ${ }^{1}$, Yuksel Oguz $^{3}$, Ahmet Erdem ${ }^{1}$ and Mehmet Erdem ${ }^{1}$ \\ ${ }^{1}$ Department of Obstetrics and Gynecology, Gazi University Faculty of Medicine, Emniyet Mahallesi, Gazeteci Yazar Muammer Yaşar \\ Bostancl Sokak, 06560 Yenimahalle/Ankara, Turkey \\ ${ }^{2}$ Department of Obstetrics and Gynecology, Zekai Tahir Burak Women's Health Education and Research Hospital, Turkey
}

\begin{abstract}
Objective: To compare the IVF outcome of patients assumed to be poor responders before their first cycle treated by microdose flare-up or GnRH antagonist protocols with patients who had a poor ovarian response after their first cycle stimulated with long GnRH protocol.

Study Design: Observational cohort study.

Place and Duration of Study: Department of Obstetrics and Gynecology, IVF Unit of Gazi University Faculty of Medicine, from September 2014 to February 2019.

Methodology: Patients treated with the first cycle of IVF and diagnosed as poor responders after ovarian stimulation were evaluated according to the treatment protocol, including microdose flare-up (Group 1: 136 patients), GnRH antagonist (Group 2: 105 patients), and long GnRH agonist (Group 3: 77 patients).

Results: Basal FSH level was significantly lower in group 3 compared to other groups $(p<0.05)$. The number of oocytes retrieved, the number of metaphase II oocytes were similar between groups, although the mean AFC was significantly higher in group 3 than in group 1 and $2(p<0.05)$. Clinical pregnancy rates per patient were higher in group $3(20.8 \%)$ than in group $1(12.5 \%)$ and group $2(13.3 \%)$, but the difference was not statistically significant $(p=0.230)$. The live birth rate per patient was statistically higher in group $3(19.5 \%)$ as compared to other groups $(8.8 \%, 9.5 \%$, respectively; $p<0.05)$.

Conclusion: Long protocol may be an option in poor responders undergoing IVF. Ovarian reserve markers are essential factors with stimulation protocol for the success of IVF in poor responder patients.
\end{abstract}

Key Words: Infertility, Ovulation Induction, Ovarian reserve, Fertilisation in-vitro, Oocyte retrieval, Pregnancy outcome, Reproductive techniques, Assisted.

How to cite this article: Demirdağ E, Akdulum MFC, Guler I, Oguz Y, Erdem A, Erdem M. IVF Outcomes of Microdose Flare-up, GnRH Antagonist, and Long Protocols in Patients having a Poor Ovarian Response in the First Treatment Cycle. J Coll Physicians Surg Pak 2021; 31(05):523-527.

\section{INTRODUCTION}

The number of cycles with poor response to ovarian stimulation (OS) protocols has been increased with the widespread use of assisted reproductive techniques (ART). In the literature, there are no precise criteria to define a poor ovarian response (POR). ESHRE (European Society of Human Reproduction and Embryology) has recently described POR when at least two features among advanced maternal age ( $\geq 40$ years) or any other risk factor for a previous POR ( $\leq 3$ oocytes by a conventional stimulation protocol); or an abnormal ovarian reserve test, i.e. $\operatorname{AFC}($ Antral follicle count $)=$ 5-7 follicles or AMH (Anti-Mullerian Hormone) $=0.5-1.1 \mathrm{ng} / \mathrm{ml} .{ }^{1}$

Correspondence to: Dr. Erhan Demirdağ, Department of Obstetrics and Gynecology, Gazi University Faculty of Medicine, Emniyet Mahallesi, Gazeteci Yazar Muammer Yaşar Bostancı Sokak, 06560 Yenimahalle/Ankara, Turkey E-mail: erhan55_gs@hotmail.com

Received: January 16, 2021; Revised: March 13, 2021;

Accepted: March 30, 2021

DOI: https://doi.org/10.29271/jcpsp.2021.05.523
A new approach for poor responders termed as "Poseidon" (Patient-oriented Strategies Encompassing Individualized Oocyte Number) stratification has been indicated recently due to the heterogeneity in definitions. This classification consists of 4 groups according to age ( $<35$ or $\geq 35$ years), ovarian reserve parameters ( $\mathrm{AFC} \geq 5$ or $<5, \mathrm{AMH} \geq 1.2$ or $<1.2$ ), and the number of retrieved oocytes after standard stimulation $(<4$ or $4-9$ oocytes). ${ }^{2}$

Several treatment protocols have been proposed to enhance POR in ART. Among many stimulation protocols (GnRH agonist, GnRH antagonist, microdose flare-up) and adjuncts (DHEA, Growth hormone, and others) for predicted poor responder, none is very effective or superior as evidence-based. ${ }^{3} \mathrm{~A}$ Cochrane review comparing different OS protocols in poor responders stated that using antagonist protocol resulted in a higher number of oocytes retrieved (NOR) compared to long protocol but a fewer NOR than flare-up protocol. ${ }^{4}$ Another recent Cochrane review has shown higher clinical pregnancy rates (CPR) and NOR in long protocol than short protocol. ${ }^{5}$ Besides this, some experts offer long protocol as the first option for poor 
responders due to better follicular synchronization. ${ }^{6}$ Thus, choosing one of these regimens is challenging because of the lack of sufficient evidence and the POR definition variations. In addition to this, most of the previous reports were about binary comparisons of these three main treatment protocols in different patients.

So, this study was aimed to evaluate the outcome of patients who were assumed to be a poor responder before stimulation and treated in their first cycle with microdose or antagonist protocols and compare their outcomes with patients who were stimulated with long $\mathrm{GnRH}$ protocol in their first cycle and had a poor response to gonadotropins with low number oocytes retrieved after stimulation.

\section{METHODOLOGY}

This observational cohort study was conducted retrospectively at the Department of Obstetrics and Gynecology, IVF Unit of Gazi University Faculty of Medicine, from September 2014 to February 2019. It was approved by the Ethics Committee of the Gazi University Faculty of Medicine. Patients applying to the IVF centre with differentetiologies of infertility and started IVF treatment were evaluated from the medical records of the hospital.

Three groups were formed according to their IVF protocol as microdose GnRH agonist (Group 1), GnRH antagonist (Group 2), and long GnRH agonist (Group 3). Patients stimulated by either microdose flare-up or GnRH antagonist protocol and anticipated as poor responders, according to their age, basal FSH, or AFC prior to stimulation, were reviewed as poor responders study groups. All patients in the microdose (Group 1) and antagonist protocol (Group 2) had the first cycle of IVF and had the number of oocytes retrieved (NOR) $\leq 5$ after ovarian stimulation. Patients treated by long luteal GnRH agonist, according to their age, basal FSH, or AFC and diagnosed as poor responders after stimulation due to the low yield of oocytes in their first IVF cycle, were evaluated as the control group. All patients in the long agonist group (Group 3) had NOR $\leq 5$ after stimulation as in study groups. When patients had $>5$ oocytes after their cycle, they were excluded from the study. Patients with a diagnosis of endocrinological disorders, including polycystic ovary syndrome, hypothyroidism or hyperprolactinemia, endometriosis, and severe male factor infertility, werealso excluded.

In Group 1, low dose OC (Desolett; Organon, Netherlands) was started on day 1 of the previous cycle for 21 days. On the second day of menstruation, $40 \mu \mathrm{g}$ subcutaneous (SC) twice daily of leuprolide acetate (Lucrin; Abbott, France) $(80 \mu \mathrm{g} /$ day) was initiated. 300-450 IU/day SC recombinant FSH (Gonal-F; Serono, Turkey) was started on the 3rd day of the cycle. Leuprolide acetate and recombinant FSH were continued until the day of hCGadministration.

In Group 2, 300-450 IU/day SC recombinant FSH was commenced on the 3rd day of the cycle. When the leading follicle reached $14 \mathrm{~mm}$ in diameter, $0.25 \mathrm{mg}$ SC cetrorelix (Cetrotide; Asta Medica, Germany) was administered daily until hCGinjection.
In Group 3, SC leuprolide acetate (1 mg) was started in the mid-luteal phase of the previous cycle and ceased when the pituitary suppression was confirmed ( $E_{2}$ level $<50 \mathrm{pg} / \mathrm{ml}$ ). Then 300-450 IU/day SC recombinant FSH was started, and leuprolide acetate was decreased to half of the initial dose $(0.5 \mathrm{mg})$. Leuprolide acetate and recombinant FSH were maintained until the day of hCG administration.

Follicle growth was followed by serial ultrasound evaluation and serum $E_{2}$ measurements to adjust the gonadotropin dose in compliance with the ovarian stimulation response. All the sonographic exams were performed by Voluson 730 Pro-machine (GE Healthcare Austria GmbH \& Co OG). 250 mcg SC choriogonadotropin alfa (Ovitrelle, Merc Serono, Italy) were used to trigger ovulation when the mean diameter of the leading follicles was observed $\geq 17-18 \mathrm{~mm}$ by ultrasonography. Transvaginal oocyte retrieval was performed 36 hours after hCG administration. ICSI procedure was carried out for all retrieved metaphase II oocytes. ET (Embryo transfer) was performed 2-3 days after oocytes retrieval forhighorgood-quality embryos (gradel [high-quality]: embryos with equal blastomere and no observed cytoplasmic fragmentation; grade II [good-quality]: embryos with equal blastomere and $<20 \%$ fragmentation of the cytoplasm) under transabdominal ultrasound guidance by using a flexible catheter (Wallace; Irvine Scientific, Santa Ana, CA).

Vaginal progesterone (P) supplementation (Crinone $8 \%$ gel, Serono) was started on all patients for luteal phase support after the transfer and continued until fetal heart activity was observed. Clinical pregnancy was diagnosed when a gestational sac or a fetus with cardiac activity was followed by ultrasonography. The live birth was defined as the delivery of a viable fetus of $\geq 23$ weeks' gestation.

Primary outcome measures were CPR and live birth rates (LBR) per patient in this study. Secondary outcome measures were the NOR, the number of mature oocytes, and estradiol levels on the day of hCG trigger. The fertilisation rate was defined as the ratio of the total number of fertilized oocytes to the total number of mature oocytes retrieved.

Data were analysed with Statistical Package for Social Sciences (SPSS, version 21.0, Statistics, 2013, Chicago, IBM, USA). Normality tests, including the Kolmogorov-Smirnov test, were used for data analyses concerning normal distribution. One-way analyses of variance (One-way ANOVA) test with Bonferroni post hoc was used to compare the mean values between stimulation protocol groups. Chi-square test was used to analyse the differences between evaluated categorical data. The fertilisation rate was compared with the Chi-square test. Continuous variables were presented as mean \pm standard deviation, and categorical data were presented as percentages. Statistical significance was defined as $p<0.05$.

\section{RESULTS}

A total of 318 patients were evaluated in this study. Group 1 had 136 (42.8\%) patients, Group 2 had 105 (33.0\%) patients, and Group 3 had 77 (24.2\%) patients. 
Table I: Comparison of basal characteristics of patients between groups.

\begin{tabular}{|c|c|c|c|c|}
\hline Variables (318 patients) & $\begin{array}{c}\text { Group } 1 \text { (Microdose) (1) } \\
(n=136)\end{array}$ & $\begin{array}{c}\text { Group } 2 \text { (Antagonist) (2) } \\
(n=105)\end{array}$ & $\begin{array}{c}\text { Group } 3 \text { (Long agonist) } \\
\text { (3) }(n=77)\end{array}$ & p-value \\
\hline Age (year) & $36.4 \pm 4.3$ & $36.1 \pm 5.3$ & $35.1 \pm 3.5$ & 0.130 \\
\hline Duration of infertility (month) & $107.7 \pm 68.5$ & $96.5 \pm 71.3$ & $100.2 \pm 62.5$ & 0.436 \\
\hline Basal FSH (mlU/ml) & $9.3 \pm 3.9(3)$ & $9.7 \pm 4.6(3)$ & $7.8 \pm 2.7(1,2)$ & 0.009 \\
\hline Antral follicle count & $4.8 \pm 1.7(3)$ & $4.7 \pm 2.0(3)$ & $5.5 \pm 1.9(1,2)$ & 0.005 \\
\hline BMI $\left(\mathrm{kg} / \mathrm{m}^{2}\right)$ & $23.1 \pm 2.3$ & $22.7 \pm 2.7$ & $23.2 \pm 2.5$ & 0.345 \\
\hline \multicolumn{4}{|l|}{ Causes of infertility $n,(\%)$} & 0.214 \\
\hline Mild male factor & $47(34.6)$ & $27(25.7)$ & $29(37.7)$ & \\
\hline Unexplained & $52(38.2)$ & $53(50.5)$ & $37(48.1)$ & \\
\hline Tubal & $22(16.2)$ & $16(15.2)$ & $7(9.1)$ & \\
\hline Mixt & $15(11)$ & $9(8.6)$ & $4(5.2)$ & \\
\hline
\end{tabular}

\begin{tabular}{|c|c|c|c|c|}
\hline $\begin{array}{l}\text { Variables } \\
\text { (318 patients) }\end{array}$ & $\begin{array}{c}\text { Group } 1 \text { (Microdose) }{ }^{(1)} \\
(n=136)\end{array}$ & $\begin{array}{c}\text { Group } 2 \text { (Antagonist) }{ }^{(2)} \\
(n=105)\end{array}$ & $\begin{array}{c}\text { Group } 3 \text { (Long } \\
\text { agonist) }{ }^{(3}(n=77)\end{array}$ & p-value \\
\hline Duration of stimulation (day) & $11.5 \pm 1.9$ & $11.0 \pm 2.5^{(1)}$ & $10.8 \pm 2.1$ & 0.054 \\
\hline Total dose of gonadotropin(IU) & $4189.5 \pm 1252.7^{(3)}$ & $3994.1 \pm 1397.9$ & $3714.7 \pm 1120.7^{(1)}$ & 0.033 \\
\hline E2 level on hCG day (pg/ml) & $1054.2 \pm 506.0$ & $933.9 \pm 427.3^{(3)}$ & $1148.0 \pm 546.9^{(2)}$ & 0.045 \\
\hline LH level on hCG day (IU/L) & $3.0 \pm 2.1$ & $3.6 \pm 3.2$ & $3.3 \pm 2.7$ & 0.475 \\
\hline Progesteron level on hCG day (ng/ml) & $0.8 \pm 0.5$ & $0.8 \pm 0.6$ & $1.0 \pm 0.7$ & 0.166 \\
\hline Number of follicle $\geq 17 \mathrm{~mm}$ on hCG day (mm) & $2.1 \pm 1.0$ & $1.9 \pm 1.0$ & $2.0 \pm 1.3$ & 0.294 \\
\hline Endometrial thickness on hCG day (mm) & $10.4 \pm 2.3$ & $10.1 \pm 2.4$ & $10.7 \pm 2.0$ & 0.179 \\
\hline Cycle cancellation rate, $\mathrm{n}(\%)$ & $12(8.8)$ & $8(7.6)$ & $7(9.1)$ & 0.924 \\
\hline Number of Oocytes retrieved & $3.2 \pm 1.3$ & $3.1 \pm 1.4$ & $3.4 \pm 1.9$ & 0.410 \\
\hline Number of MII Oocytes & $2.7 \pm 1.2$ & $2.5 \pm 1.3$ & $2.9 \pm 1.4$ & 0.138 \\
\hline Fertilization rates, $\mathrm{n}$ of PN (\%) & $262(70.6)$ & $183(69.1)$ & $150(67)$ & 0.645 \\
\hline Number of transferred embryos & $1.8 \pm 0.8$ & $1.7 \pm 0.7$ & $1.6 \pm 0.7$ & 0.104 \\
\hline Clinical pregnancy rate, per patient, n (\%) & $17(12.5)$ & $14(13.3)$ & $16(20.8)$ & 0.230 \\
\hline Live birth rate, per patient, $\mathrm{n}(\%)$ & $12(8.8)$ & $10(9.5)$ & $15(19.5)$ & 0.047 \\
\hline
\end{tabular}

Basal characteristics of groups were shown in Table I. The mean AFC was significantly higher in group $3(5.5 \pm 1.9)$ than group $1(4.8 \pm 1.7)$ and group $2(4.7 \pm 2.0, p<0.05)$, and the mean basal FSH level was significantly lower in Group $3(7.8 \pm 2.7)$ compared to group 1 and 2 (9.3 \pm 3.9 , $9.7 \pm 4.6$, respectively, $p<0.05)$.

The comparison of ovarian stimulation parameters between groups was given in Table II. Patients in Group 1 used a significantly higher total dose of gonadotropins (4189.5 \pm $1252.7)$ as compared to group $3(3714.7 \pm 1120.7, p<0.05)$. The mean estradiol level on the day of hCG trigger was significantly higher in Group 3 (1148.0 \pm 546.9) as compared to only Group $2(933.9 \pm 427.3, p<0.05)$. The mean total NOR, number of metaphase II oocytes, and the number of transferred embryos were similar among groups. Fertilisation rates were not different between groups (70.6\%, $69.1 \%, 67 \%$, respectively, $p=0.645$ ).

Clinical pregnancy was achieved in 47 of 318 patients (14.8\%) for all Groups. In Group 3, the CPR was higher than
Group 1 and 2, but the difference was not statistically significant $(20.8 \%, 12.5 \%, 13.3 \%$, respectively; $p=0.230)$. Overall live birth was reported in 37 of 318 patients (11.6\%). LBR per patient was statistically higher in Group 3 compared to Group 1 and 2 (19.5\%, 8.8\%, 9.5\%, respectively; $p<0.05$ ).

\section{DISCUSSION}

In this study, GnRH antagonist, microdose flare-up, and long luteal protocols were compared for IVF cycles of poor responders. The key finding was that although the NOR and the number of metaphase II oocytes were comparable among Groups, the LBR was statistically higher in the long luteal group than microdose flare-up and GnRH antagonist protocol. Based on the theory of avoiding further suppression of endogen gonadotropins, GnRH antagonist and microdose flare-up protocols were thought to be superior to long luteal protocol. These ovarian stimulation protocols were mostly compared with each other in binary. ${ }^{7-9}$ However, there is limited data for IVF outcomes of these protocols concurrently with long protocol in poor responders 
in a cohort. ${ }^{10}$ Moreover, literature consisted of many studies with different results in which these three protocols were compared in binary.

In the previous studies comparing microdose flare-up with long protocol, while some of them reported significantly increased pregnancy rates (PR) and decreased cancellation rates $(C R),{ }^{11,12}$ another reported higher CR with similar PR with microdose protocol. ${ }^{13}$ Oocyte numbers were found to be similar along with no difference in the reproductive outcomes. ${ }^{14}$ Conversely, Cochrane review revealed significantly higher CPR and NOR in long protocol than short flare-up protocol, ${ }^{5}$ although the outcomes of short flare protocols did not reflect microdose flare-up protocols'.

Comparisons of microdose flare-up and $\mathrm{GnRH}$ antagonist protocols also have inconsistent results in the literature. Many of them reported similar NOR and PR in both groups. ${ }^{7,8,15}$ The higher number of metaphase II oocytes and LBR with similar CPR was also reported in the microdose protocol, respectively. ${ }^{16,17}$ In contrast to the above, some found that antagonist protocol was superior to microdose flare-up regarding $\mathrm{OR}^{9}{ }^{9}$ and the number of metaphase II oocytes retrieved in which estrogen priming was also performed with $\mathrm{GnRH}$ antagonists. ${ }^{18}$ In this study, NOR, CPR, and LBR were not different between these two groups, which was in line with previous reports.

When GnRH antagonist and the long protocol was compared in poor responders, higher NOR, implantation and PR were reported in the $\mathrm{GnRH}$ antagonists. ${ }^{4,19}$ On the other hand, others found that long agonist protocol improved NOR and CPR compared to the GnRH antagonist group in poor responders. ${ }^{20}$ However, a recent meta-analysis has reported similar NOR, CPR, and LBR among these two groups. ${ }^{21}$

This study found higher LBR in the long agonist group than in other two protocols. All these protocols have also been assessed recently by Sunkara et al.; and non-significantly higher OPR has been found in the GnRH antagonist group than in others. ${ }^{10}$ The small sample size may lead to this nonsignificant difference, as stated by the authors. In this study, higher pregnancy rates in the long $\mathrm{GnRH}$ group could also be the relatively good prognosis of these patients, although the mean age was similar between Groups. The long protocol group included patients with the lowest FSH level and the highest AFC on the 3rd day of the cycle, which may contribute to these good results. The long protocol may also be associated with more synchronized follicle development, as proposed by some authors previously. ${ }^{6}$ The long agonist group may also probably represent Poseidon Group 1 or 2, which has been described in recent years for poor responder patients. In poor responders with a good prognosis like Poseidon Group 1 and 2, prognostic factors such as ovarian reserve parameters seem crucial on IVF outcomes in addition to the stimulation protocol as in this study. Although the study population's homogeneity could not be provided to be the best, three stimulation protocols were concurrently evaluated in our research. This results indicate supporting the long agonist use in the poor responders. Since the microdose flare-up protocol did not change the outcomes significantly, its use has not been recommended anymore in the recent ESHRE guidelines for ovarian stimulation. ${ }^{3}$

\section{CONCLUSION}

In poor responders with relatively good ovarian reserve markers before stimulation, ovarian stimulation with long protocol might positively affect pregnancy outcomes in IVF cycles.

\section{ACKNOWLEDGEMENT:}

We thank Gazi University for the support of this study.

\section{ETHICAL APPROVAL:}

This observational cohort study was approved by the Ethics Committee of the Gazi University Faculty of Medicine.

\section{PATIENTS' CONSENT:}

Informed consents were obtained from all patients.

\section{CONFLICT OF INTEREST:}

The authors declared no conflict of interest.

\section{AUTHORS' CONTRIBUTION:}

ED: Drafting the work or revising it critically for important intellectual content and interpretation of data for the work. MFCA, YO: Data acquisition.

IG: Substantial contributions to the conception or design of the work.

$A E$ : Substantial contributions to the conception.

ME: Final approval of the version to be published, and revising it critically for important intellectual content.

\section{REFERENCES}

1. Ferraretti A, La Marca A, Fauser B, Tarlatzis B, Nargund G, Gianaroli $L$, et al. ESHRE consensus on the definition of poor response'to ovarian stimulation for in vitro fertilization: The bologna criteria. Hum Reprod 2011; 26(7):1616-24. doi: 10.1093/humrep/der092.

2. Humaidan P, Alviggi C, Fischer R, Esteves SC. The novel POSEIDON stratification of 'Low prognosis patients in Assisted Reproductive Technology'and its proposed marker of successful outcome. F1000 Res 2016; 5:2911 doi: 10.12688/f1000research.

3. Ovarian Stimulation TEGGo, Bosch E, Broer S, Griesinger G, Grynberg M, Humaidan $P$, et al. ESHRE guideline: ovarian stimulation for IVF/ICSI. Hum Reprod Open 2020; 2020(2):hoaa009. doi: 10.1093/hropen/hoaa009. 
4. Pandian Z, McTavish AR, Aucott L, Hamilton MP, Bhattacharya S. Interventions for'poor responders' to controlled ovarian hyper stimulation $(\mathrm{COH})$ in in-vitro fertilisation (IVF). Cochrane Database Syst Rev 2010; (1): CD004379.

5. Siristatidis CS, Gibreel A, Basios G, Maheshwari A, Bhattacharya S. Gonadotrophin-releasing hormone agonist protocols for pituitary suppression in assisted reproduction. Cochrane Database Syst Rev 2015; (11):CD006919. doi: 10.1002/14651858.CD006919.pub4.

6. Haahr T, Dosouto C, Alviggi C, Esteves SC, Humaidan P. Management strategies for POSEIDON groups 3 and 4 . Front Endocrinol 2019; 10:614. doi: 10.3389/fendo.2019. 00614.

7. Kahraman K, Berker B, Atabekoglu CS, Sonmezer M, Cetinkaya E, Aytac R, et al. Microdose gonadotropinreleasing hormone agonist flare-up protocol versus multiple dose gonadotropin-releasing hormone antagonist protocol in poor responders undergoing intracytoplasmic sperm injection-embryo transfer cycle. Fertil Steril 2009; 91(6):2437-44. doi: 10.1016/j.fertnstert.2008.03.057.

8. Schmidt DW, Bremner T, Orris JJ, Maier DB, Benadiva CA, Nulsen JC. A randomised prospective study of microdose leuprolide versus ganirelix in in vitro fertilization cycles for poor responders. Fertil Steril 2005; 83(5):1568-71. doi: 10.1016/j.fertnstert.2004.10.053.

9. Lainas TG, Sfontouris IA, Papanikolaou EG, Zorzovilis JZ, Petsas GK, Lainas GT, et al. Flexible GnRH antagonist versus flare-up $\mathrm{GnRH}$ agonist protocol in poor responders treated by IVF: a randomised controlled trial. Hum Reprod 2008; 23(6):1355-8. doi: 10.1093/humrep/den107.

10. Sunkara SK, Coomarasamy A, Faris R, Braude P, Khalaf $Y$. Long gonadotropin-releasing hormone agonist versus short agonist versus antagonist regimens in poor responders undergoing in vitro fertilization: A randomised controlled trial. Fertil Steril 2014; 101(1):147-53. doi: 10.1016/ j.fertnstert.2013.09.035.

11. Surrey ES, JoAnn Bower RNC N, Hill DM, Juliana Ramsey RNC N, Surrey MW. Clinical and endocrine effects of a microdose $\mathrm{GnRH}$ agonist flare regimen administered to poor responders who are undergoing in vitro fertilisation. Fertil Steril 1998; 69(3):419-24. doi: 10.1016/s0015-0282 (97)00575-x.

12. Toth TL, Awwad JT, Veeck LL, Jones Jr H, Muasher SJ. Suppression and flare regimens of gonadotropin-releasing hormone agonist. Use in women with different basal gonadotropin values in an in vitro fertilization program. J
Reprod Med 1996; 41(5):321-6.

13. Leondires MP, Escalpes M, Segars JH, Scott Jr RT, Miller BT. Microdose follicular phase gonadotropin-releasing hormone agonists (GnRH-a) compared with luteal phase GnRH-a for ovarian stimulation at in vitro fertilization. Fertil Steril 1999; 72(6):1018-23. doi: 10.1016/s0015-0282(99) 00423-9.

14. Chatillon-Boissier K, Genod A, Denis-Belicard E, Felloni B, Chene $G$, Seffert $P$, et al. Prospective randomised study of long versus short agonist protocol with poor responder patients during in vitro fertilization. Gynecol Obstet Fertil 2012; 40(11):652-7. doi: 10.1016/j.gyobfe.2011.08.025.

15. Merviel P, Cabry-Goubet R, Lourdel E, Devaux A, BelhadriMansouri N, Copin $\mathrm{H}$, et al. Comparative prospective study of 2 ovarian stimulation protocols in poor responders: Effect on implantation rate and ongoing pregnancy. Reprod Health 2015; 12(1):1-7. doi: 10.1186/s12978-015-0039-2.

16. Boza A, Cakar E, Boza B, Api M, Kayatas S, Sofuoglu K. Microdose flare-up gonadotropin-releasing hormone ( $\mathrm{GnRH}$ ) agonist versus $\mathrm{GnRH}$ antagonist protocols in poor ovarian responders undergoing intracytoplasmic sperm injection. J Reprod Infertil 2016; 17(3):163.

17. Ghaffari F, Jahangiri N, Madani T, Khodabakhshi S, Chehrazi M. Randomized controlled trial of gonadotropin-releasing hormone agonist microdose flare-up versus flare-up among poor responders undergoing intracytoplasmic sperm injection. Int J Gynaecol Obstet 2020; 148(1):59-64. doi: 10.1002/ijgo.12988.

18. Davar R, Neghab N, Naghshineh E. Pregnancy outcome in delayed start antagonist versus microdose flare $\mathrm{GnRH}$ agonist protocol in poor responders undergoing IVF/ICSI: An RCT. Int J Reprod Biomed 2018; 16(4):255.

19. Cheung LP, Lam PM, Lok IH, Chiu TTY, Yeung SY, Tjer CC, et al. GnRH antagonist versus long $\mathrm{GnRH}$ agonist protocol in poor responders undergoing IVF: A randomised controlled trial. Hum Reprod 2005; 20(3):616-21. doi: 10.1093/hum rep/deh668.

20. Prapas Y, Petousis S, Dagklis T, Panagiotidis Y, Papatheodorou A, Assunta I, et al. GnRH antagonist versus long GnRH agonist protocol in poor IVF responders: A randomised clinical trial. Eur J Obstet Gynecol Reprod Biol 2013; 166(1):43-6. doi: 10.1016/j.ejogrb.2012.09.008.

21. Lambalk C, Banga F, Huirne J, Toftager M, Pinborg A, Homburg R, et al. GnRH antagonist versus long agonist protocols in IVF: a systematic review and meta-analysis accounting for patient type. Hum Reprod Update 2017; 23(5):560-79. doi: 10.1093/humupd/dm×017. 